\title{
ANÁLISE DO TEOR DE FLÚOR NAS ÁGUAS DE ABASTECIMENTO PÚBLICO DE PONTA GROSSA-PR: DEZ MESES DE HETEROCONTROLE
}

\section{ANALYSIS OF FLUORIDE LEVELS IN THE PUBLIC WATER SUPPLIES IN PONTA GROSSA-PR: A TEN-MONTH EXTERNAL CONTROL}

\author{
Denise Stadler Wambier ${ }^{*}$, Márcia Helena Baldani Pinto ${ }^{1}$, \\ Alberto Estefano Guilherme Kloth², Maria Lúcia Vetorazzi³, \\ Rafael Gomes Ditterich ${ }^{4}$, Deyvis Kolodziyczyk de Oliveira ${ }^{5}$ \\ 1* Autor para contato: Universidade Estadual de Ponta Grossa - UEPG. Departamento de \\ Odontologia, Campus em Uvaranas, Ponta Grossa, PR, Brasil; (42) 3220-3104; \\ e-mail:dswambier@yahoo.com.br \\ 2 Universidade Estadual de Ponta Grossa - UEPG, Departamento de Biologia Geral, Campus \\ em Uvaranas, Ponta Grossa, PR \\ 3 Centro de Ensino Superior dos Campos Gerais - CESCAGE, Ponta Grossa, PR \\ 4 Universidade Federal do Paraná - UFPR, Curitiba, PR \\ 5 Universidade Estadual de Ponta Grossa - UEPG, Departamento de Odontologia, Campus \\ em Uvaranas, $\mathrm{PIBIC} / \mathrm{CNPq} / \mathrm{UEPG}$
}

Recebido para publicação em 02/05/2007

Aceito para publicação em 15/08/2007

\section{RESUMO}

Este estudo objetivou monitorar, durante dez meses, a concentração e regularidade dos teores de fluoretos nas águas de abastecimento público do município de Ponta Grossa-PR. Foram realizadas coletas mensais de água nos reservatórios urbanos das Estações de Tratamento da SANEPAR, todas no mesmo horário e em datas previamente estabelecidas, durante o período de outubro de 2006 a julho de 2007. A con-centração de flúor presente nas amostras de água foi determinada pelo método colorimétrico. Uma vez analisadas, estas foram classificadas como aceitáveis ou não, de acordo com a concentração de flúor encontrada. Das amostras analisadas, pequena parte apresentou teores aceitáveis de fluoretos. As demais mostraram teores inaceitáveis, sendo que algumas se apresentavam-se subfluoretadas $(<0,55 \mathrm{mg} / \mathrm{L})$ e a maior parte superfluoretadas $(>0,85 \mathrm{mg} / \mathrm{L})$. Verificou-se uma inconstância nos teores de fluoretos encontrados nas amostras, predominando o número de amostras coletadas com valores acima dos aceitáveis para o município. Os resultados indicam a urgência de um programa de heterocontrole em Ponta Grossa-PR.

Palavras-chave: flúor, fluoretação da água, análise da água, abastecimento de água 


\begin{abstract}
This study aimed to carry out a ten-month assessment of the concentration and regularity of fluoride levels in the public water supplies in the municipality of Ponta Grossa-PR. Water samples were collected monthly in the urban reservoirs of the SANEPAR water treatment station, all at the same time of day and on previously established dates, from October 2006 through Jule 2007. The fluoride levels in the water samples were determined by the colorimetric method. The samples were analyzed, then classified as acceptable or not, according to fluoride concentrations. Only a small number of the samples presented acceptable fluoride levels. Most of the samples were underfluorinated $(<0,55 \mathrm{mg} / \mathrm{L})$ or overfluorinated $(>0,85 \mathrm{mg} / \mathrm{L})$. Results evinced inconstancy in fluoride levels, most of them with values above the ones acceptable for the municipality. The results indicate the need of an urgent external water control program in Ponta Grossa-PR.
\end{abstract}

Key words: fluorine, fluoridation, water analysis, public water supply

\section{Introdução}

O uso de água fluoretada é um método que beneficia a população, contribuindo com a redução de cárie dentária, porém quantidades adequadas devem ser administradas para que se alcance o benefício esperado sem o risco do desenvolvimento de alterações dentárias.

Atualmente, os indivíduos estão sendo expostos a várias fontes de fluoretos (dentifrícios, enxagüatórios bucais, suplementos fluoretados, fórmulas infantis, etc.), distribuídos de forma indistinta, tanto para áreas com água de abastecimento fluoretada como não fluoretada (Mascarenhas, 2000). Baseados nesta nova realidade, evidências sugerem que a múltipla exposição a estes diferentes veículos contribuiu para o declínio da cárie, ao mesmo tempo em que se constatou aumento da fluorose dentária (Moysés et al., 2002; Frazão et al., 2004).

No que diz respeito aos riscos de fluorose, Bighetti (2005) estudou os fatores associados com esta alteração em área com água fluoretada e verificou risco significativo quando os adolescentes usaram dentifrício fluoretado nos seis primeiros anos de vida e eram responsáveis pela sua colocação na escova. Salientou que os serviços de saúde devem planejar ações de educação, reforçando o uso adequado de dentifrícios, além de manter a política de vigilância sanitária dirigida às múltiplas fontes de flúor.
No município de Ponta Grossa, Ditterich (2005) verificou em 473 escolares de 12 anos, a prevalência de fluorose dentária por meio do índice de Dean (OMS, 1999) . Constatou que 19,65\% dos examinados apresentavam manchas fluoróticas, que variaram de grau muito leve a moderado. Neste mesmo município, Kozlowski (2004) coletou amostras de água de abastecimento público em seis pontos da cidade, e verificou que uma das amostras apresentou baixo teor de flúor $(<0,04 \mathrm{mgF} / \mathrm{L})$, enquanto que, nas outras cinco, as concentrações de flúor variaram de 0,94 a 1,17 mgF/L.

A lei n ${ }^{\circ} .6050$, de 24 de maio de 1974, dispõe sobre a obrigatoriedade da fluoretação das águas de abastecimento público em localidades onde exista Estação de Tratamento de Água (ETA). Assim como a Portaria do Ministério da Saúde nº . 635, de 25 de dezembro de 1975, aprova e determina normas e padrões a serem seguidos para a correta fluoretação das águas, desde a concentração a ser utilizada, de acordo com as médias das temperaturas máximas anuais de cada região, até os compostos recomendados (BRASIL, 1974, 1975; Kozlowski; Pereira, 2003; Narvai, 2000; Schneider Filho et al., 1992).

A opção brasileira pela fluoretação das águas foi em decorrência de sua extensão territorial, e também ao fato de que o método possibilita estender os benefícios do flúor a toda a população, independente da condição socio-econômica (Kozlowski; Pereira, 2003). 
Quanto a este aspecto, a fluoretação das águas tem sido considerada como o método mais equânime de controle de cárie, uma vez que é capaz de reduzir o impacto das desigualdades socioeconômicas na experiência desta doença (Slade et al., 1996; Jones et al., 1997; Jones; Worthington, 2000; Burt 2002; Baldani et al., 2004). No Paraná, a fluoretação das águas teve início em Curitiba no ano de 1958.

Diversos estudos evidenciaram que o nível ótimo, em termos de risco/benefício, de fluoretos nas águas de abastecimento público para a redução da doença cárie, encontra-se entre 0,7 a 1,2 mgF/L, de acordo com a temperatura média anual de cada localidade (Pinto, 1992; Kozlowski; Pereira, 2003; Murray, 1992; OMS, 1994).

Segundo Pinto (1992), quando a dosagem é excessiva e administrada por longos períodos de tempo, pode ocorrer fluorose dentária já a partir de 1,2 ppm $\mathrm{F}$ diários, embora somente nos limites entre 2 à $8 \mathrm{ppm}$ F se constatem níveis moderados ou severos desta doença.

Nesta linha de raciocínio, os mecanismos de vigilância são fundamentais para a melhoria da qualidade da água a ser fornecida à população, sendo, portanto, indispensável à ação correta da empresa responsável pelo seu tratamento e fluoretação (Cury, 1992; Maia et al., 2003; Narvai, 2001).

Para se compreender o sistema de monitoramento da fluoretação das águas de abastecimento é preciso conhecer a diferença entre os termos: Controle Operacional e Sistema de Vigilância ou Heterocontrole.

Controle Operacional refere-se aos mecanismos de controle sobre o processo de fluoretação nas estações de tratamento das águas (ETAs), sendo executado pela própria empresa de saneamento, no sentido de prevenir ou corrigir eventuais problemas na operação do sistema. Para tanto, esse controle deve incluir várias mensurações diárias, com o estabelecimento de valores médios, máximos e mínimos em cada dia, permitindo avaliações constantes em cada ETA, adutoras e distribuidoras (Dantas et al., 1996; Schneider et al., 1992).

Os Sistemas de Vigilância ou Heterocontrole, por outro lado, tem o objetivo de acompanhar a execução da medida, a partir dos seus efeitos na água oferecida ao consumidor, em diferentes localidades do município, por meio do exame de amostras coletadas em torneiras, ou seja, na ponta da rede de abastecimento. Esta vigilância é realizada por instituições públicas ou entidades privadas, distintas da empresa responsável pela fluoretação (Schneider et al., 1992). Este mecanismo de vigilância favorece o controle social do uso de fluoretos nas águas, permitindo a divulgação da situação encontrada à sociedade e as suas organizações civis (Dantas et al., 1996).

Estudos de heterocontrole realizados em VitóriaES e no Rio de Janeiro-RJ revelaram, nas amostras analisadas, concentrações inferiores à $0,7 \mathrm{mgF} / \mathrm{L}$. Este fato poderia determinar efeito cariostático menos efetivo (Ferreira et al., 1999; Modesto et al., 1999).

Em Pelotas-RS, foram encontrados locais com concentrações abaixo de $0,7 \mathrm{mgF} / \mathrm{L}$, bem como outros com teores de flúor superiores à $1,2 \mathrm{mgF} / \mathrm{L}$, o que poderia levar a uma maior prevalência de fluorose, desde que a ingestão de água ocorresse no período préeruptivo e de forma contínua (Lima et al., 2004).

Pesquisa realizada em 40 municípios situados na região noroeste do estado de São Paulo objetivou verificar se a adição de flúor ocorria de forma contínua e se os teores adicionados encontravam-se dentro dos parâmetros recomendados. Amostras com 0,6 a 0,8 $\mathrm{mg} \mathrm{F} / \mathrm{L}$ foram consideradas aceitáveis. Nos 38 municípios que enviaram as amostras regularmente nos seis meses do estudo, foi coletada água em 144 pontos, perfazendo um total de 864 amostras. Destas, $61,8 \%$ foram classificadas como inaceitáveis. As concentrações de flúor variavam entre os pontos e no mesmo ponto ao longo do período em $78,7 \%$ dos municípios. A maioria deles não mantinha controle adequado sobre os níveis de flúor em sua água, pois a adição era descontínua e com teores fora dos parâmetros recomendados (Saliba et al., 2006).

Após doze meses de monitoramento dos níveis de flúor da água de abastecimento público de Lages$\mathrm{SC}$, foram verificados teores inadequados em $45,8 \%$ das amostras, sendo que houve predomínio de locais com excesso de fluoretos (35,8 por cento). A concentração estava adequada em $54,2 \%$ das unidades amostrais (Toassi et al., 2007).

A análise das concentrações de flúor nas águas de abastecimento público em municípios do Piauí (Teresina, Floriano e Parnaíba) demonstrou que o teor de flúor na maioria da amostras coletadas ficou abaixo da concentração ideal e apenas 4,3\% apresentaram valores aceitáveis $(0,60$ a $0,80 \mathrm{mgF} / \mathrm{L})$. Por cidade, $\mathrm{o}$ percentual de amostras aceitáveis foi de 7,8\%, 4,7\% e $0,5 \%$ para Teresina, Floriano e Parnaíba, respectiva- 
mente. As concentrações de flúor na água de abastecimento público das cidades avaliadas estavam abaixo do ideal (Silva et al., 2007).

Verifica-se do exposto que o melhor benefício da fluoretação com o menor risco de efeitos negativos depende de vigilância externa, ainda não instituída no município de Ponta Grossa.

\section{Materiais e métodos}

Esta pesquisa, longitudinal foi desenvolvida na cidade de Ponta Grossa, localizada na região Centro Sul do estado do Paraná (Brasil), e que possuía, em 2006, uma população estimada de 304.973 habitantes (IBGE, 2007). Sua temperatura média anual situa-se entre 18 e $19^{\circ} \mathrm{C}$, variando desde uma média de $14^{\circ} \mathrm{C}$ no inverno até $20^{\circ} \mathrm{C}$ no verão.

O município conta com o benefício da fluoretação das águas desde 1985, sendo que a concentração de flúor nos dias atuais tem variado de 0,6 a $1,1 \mathrm{mgF} / \mathrm{L}$, atendendo à legislação vigente.

Existem no município duas estações de tratamento (ETAs), abastecidas por água captada da Represa de Alagados (ETAI) e do Rio Pitangui (ETAII). As águas provenientes das duas ETAS se misturam após o tratamento, seguindo para cinco reservatórios, a partir dos quais ocorre a distribuição para a população. Para este estudo, o mapeamento dos pontos de coleta foi definido a partir da distribuição da água tratada nas duas ETAs, com auxílio de mapa dos bairros da cidade. A escolha dos locais foi feita de forma abrangente para incluir todas as regiões da cidade, correspondendo aos pontos finais da rede de distribuição. Foram utilizados os critérios estabelecidos pelo Laboratório de Vigilância Sanitária de flúor da Facul- dade de Odontologia da Univali, Itajaí, SC (Lima et al. 2004) que recomendam o mínimo de 15 pontos de coleta para cidades com mais de duzentos mil habitantes servidos por água tratada.

Frascos de $50 \mathrm{ml}$ foram utilizados para a coleta das amostras de água retiradas diretamente de torneiras, e dos cinco reservatórios. Todas as amostras foram coletadas mensalmente e de maneira uniforme ao longo do período de dez meses. Foram incluídos vinte pontos diferentes, sendo cinco referentes aos reservatórios de água da SANEPAR e mais três pontos abastecidos por cada um. O objetivo desse procedimento foi verificar possíveis perdas de concentração de flúor ao longo da rede de distribuição.

As amostras foram armazenadas em geladeira e depois enviadas para as análises no laboratório de Higiene Social e Análise de Água do Departamento de Biologia da Universidade Estadual de Ponta Grossa. Algumas amostras foram analisadas em duplicata (10\%) para se verificar a concordância intra-examinador, e o mesmo número de contra-provas foram encaminhadas para o laboratório de análise de água da Universidade Católica do Paraná, para se obter a concordância inter-examinador. Utilizou-se o método de mensuração colorimétrico. O prazo de armazenamento não ultrapassou 30 dias, segundo Prado et al. (1992), no período em torno de 150 dias, os teores de flúor não se alteram.

Os dados obtidos foram analisados pelo programa SPSS 15.0 for Windows, utilizando-se de estatística descritiva, gráficos e tabela para apresentação dos resultados. Para fins de análise as amostras foram distribuídas em cinco grupos, correspondentes aos reservatórios de origem. Estas foram identificadas como aceitáveis ou não de acordo com a classificação proposta por Ramires et al. (2006), tabela 1.

Tabela 1 - Classificação das amostras de acordo com a concentração de flúor (mg/L).

\begin{tabular}{lc}
\hline Concentração de flúor & Classificação \\
\hline 0 a 0,54 & Inaceitável \\
$\mathbf{0 , 5 5}$ & Teor mínimo aceitável \\
0,55 a 0,64 & Subfluoretada \\
0,65 a 0,74 & Ótima \\
0,75 a 0,84 & Superfluoretada \\
$\mathbf{0 , 8 4}$ & Teor máximo aceitável \\
0,85 a 1,14 & Inadequada \\
$>1,14$ & Inaceitável \\
\hline
\end{tabular}




\section{Resultados}

Os resultados desta pesquisa são apresentados na tabela 2 e nas figuras 1 e 2 . Observa-se que, com exceção do primeiro mês de coleta (c1), o número de amostras classificadas como aceitáveis foi reduzido (figura 1). Verifica-se ainda que a quantidade de amostras com teores de flúor superiores ao máximo aceitável aumenta com o passar do tempo, sendo que o maior número daquelas com teores de flúor acima de 1,14 $\mathrm{mgF} / \mathrm{L}$ ocorrem justamente nos meses mais quentes.
Esta tendência se repete na figura 2, na qual pode-se observar a variação temporal das médias dos teores de flúor encontrados nas amostras.

Ao analisar as amostras agrupadas segundo o reservatório de origem (tabela 2), nota-se que apenas um deles apresentou mais de $50 \%$ de suas amostras com teores aceitáveis de flúor (R5). Todos os demais apresentaram elevadas proporções de amostras inaceitáveis, chegando a $80 \%$ no reservatório imediatamente próximo das ETAs.

Tabela 2 - Distribuição proporcional das amostras de água de abastecimento público de Ponta Grossa, coletadas no período de setembro de 2006 a junho de 2007.

\begin{tabular}{|c|c|c|c|c|c|c|c|c|c|c|c|c|}
\hline \multirow[b]{3}{*}{ Reservatório } & \multirow{2}{*}{\multicolumn{2}{|c|}{ Aceitável }} & \multicolumn{8}{|c|}{ Inaceitável } & \multirow{2}{*}{\multicolumn{2}{|c|}{ Total de amostras }} \\
\hline & & & \multicolumn{2}{|c|}{$<0,55$} & \multicolumn{2}{|c|}{$>0,84$} & \multicolumn{2}{|c|}{$>1,14$} & \multicolumn{2}{|c|}{ Total } & & \\
\hline & $\mathrm{N}$ & $\%$ & $\mathrm{n}$ & $\%$ & $\mathrm{n}$ & $\%$ & $\mathrm{n}$ & $\%$ & $\mathrm{n}$ & $\% *$ & $\mathrm{n}$ & $\%$ \\
\hline $\mathrm{R} 1$ & 2 & 20,0 & 3 & 30,0 & 4 & 40,0 & 1 & 10,0 & 8 & 80,0 & 10 & 100,0 \\
\hline $\mathrm{R} 2$ & 10 & 34,5 & 4 & 13,8 & 9 & 31,0 & 6 & 20,7 & 19 & 65,5 & 29 & 100,0 \\
\hline R3 & 7 & 21,9 & 3 & 9,4 & 19 & 59,4 & 3 & 9,4 & 25 & 78,1 & 32 & 100,0 \\
\hline $\mathrm{R} 4$ & 12 & 31,6 & 1 & 2,6 & 18 & 47,4 & 7 & 18,4 & 26 & 68,4 & 38 & 100,0 \\
\hline $\mathrm{R} 5$ & 11 & 61,1 & 1 & 5,5 & 6 & 33,4 & 0 & 0 & 7 & 38,9 & 18 & 100,0 \\
\hline Total & 42 & 33,1 & 12 & 9,5 & 56 & 44,1 & 17 & 13,4 & 85 & 66,9 & 127 & 100,0 \\
\hline
\end{tabular}

$\mathrm{R}=$ Sanepar

R2 = Futsal (incluindo os pontos ab\stecidos - Campus da UEPG, Borsato, Santa Mônica, Vila Ruibine)

R3 = Suíço (+ Rio Verde, Cescage (Ronda), Cipa, Cará-Cará)

R4 = Centro ( $\mathrm{ABO}$, Posto Rio Branco, Tozetto)

R5 = Los Angeles (+ Jardim Esplanada, Shangri-lá)

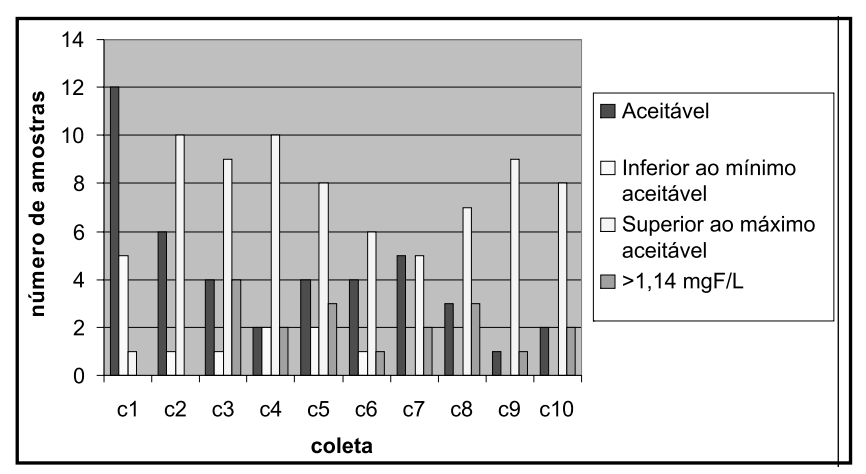

Figura 1 - Distribuição das amostras obtidas em cada coleta (c1 a c10), segundo classificação em aceitáveis ou não.

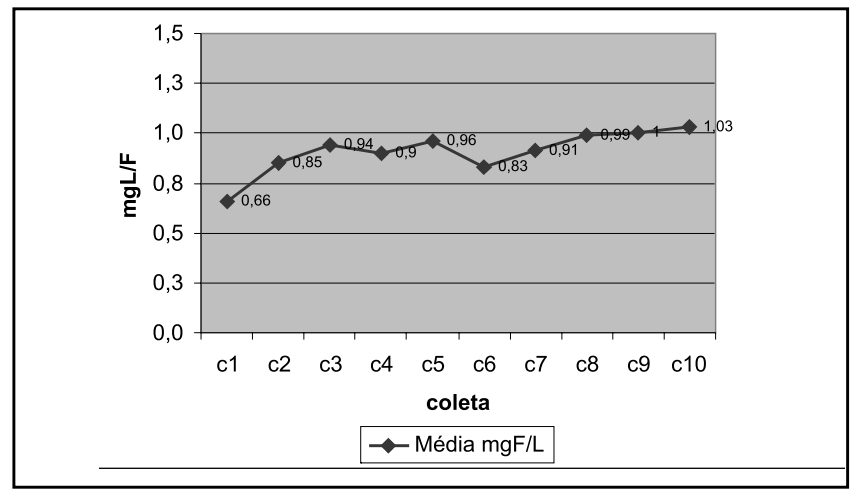

Figura 2 - Média dos teores de fluoretos encontrados nas amostras de água de abastecimento público de Ponta Grossa (setembro / 2006 - julho / 2007). 


\section{Discussão}

Levando em conta o que é considerado ideal para o município $(0,7 \mathrm{mgF} / \mathrm{L})$, os resultados desta pesquisa não foram favoráveis, pois $2 / 3$ das amostras tiveram concentrações inaceitáveis de fluoreto frente a $1 / 3$ que foi considerado aceitável. Entre as amostras com valores inaceitáveis de fluoreto, a maioria estava superfluoretada e as demais hipofluoretadas. Além disso, as concentrações de flúor variaram de $0,3 \mathrm{mgL} /$ $\mathrm{F}$ até $1,47 \mathrm{mgL} / \mathrm{F}$, sendo que valores mais elevados foram encontrados nos meses de dezembro, janeiro e fevereiro, todos eles no Reservatório Futsal. Considerando-se a legislação vigente (Portaria MS 1469, de 20 de dezembro de 2000), todas as amostras apresentaram-se com teores dentro dos parâmetros permitidos de até $1,5 \mathrm{mgF} / \mathrm{L}$. No entanto, deve-se considerar que a própria Portaria MS 1469 remete à necessidade de se adequar os valores de cada município à legislação específica sobre a fluoretação de águas, a qual recomenda que os teores de fluoretos devem ser calculados de acordo com a média das máximas temperaturas da região, medidas durante um período de cinco anos (Portaria MS 635, de 25 de dezembro de 1975). Segundo esta legislação, a concentração ideal de flúor nas águas de abastecimento de Ponta Grossa deve estar em $0,73 \mathrm{mgF} / \mathrm{L}$.

É importante destacar que altas concentrações de flúor na água podem elevar os níveis de fluorose na população, especialmente nas estações mais quentes onde o consumo de água é maior. Para que a fluoretação das águas tenha máxima eficiência, os níveis de flúor devem estar dentro do chamado "nível ótimo"e de forma ininterrupta por longos períodos. Enquanto a subdosagem não traz benefícios, a sobredosagem, por tempo continuado, durante o período de formação dos dentes, associa-se à ocorrência de fluorose (Toassi et al., 2007). Este fato indica a necessidade do controle da fluoretação para que ela alcance os benefícios esperados de redução da doença cárie, sem qualquer risco de comprometimento estético ocasionado pela presença de fluorose.

A freqüente oscilação nos níveis de flúor, com valores mais altos ou mais baixos em relação aos valores ideais para cada região, tem sido destacada em pesquisas conduzidas nas diferentes cidades brasileiras
(Ferreira et al., 1999; Modesto et al., 1999; Lima et al., 2004; Saliba et al., 2006; Toassi et al., 2007; Silva et al., 2007).

Os resultados verificados no presente estudo, com a maioria das amostras consideradas inaceitáveis, foram semelhantes aos obtidos por Saliba et al., 2006. Podem ser considerados menos favoráveis quando comparado ao estudo de Toassi et al., 2007, que obteve mais da metade das amostras com concentrações adequadas de fluoreto. Contudo, os resultados não foram tão diferentes ao analisar os altos teores de flúor em Ponta Grossa e em Lages (Toassi et al., 2007).

Resultados preocupantes estão registrados na pesquisa de Silva et al., 2007, onde somente poucas amostras apresentaram valores aceitáveis, com predomínio de concentrações inferiores ao ideal para as cidades estudadas (Teresina, Floriano e Paraíba). Ao contrário, nesta pesquisa houve predomínio de valores superiores ao ideal, sendo que a maioria das amostras estavam superfluoretadas. Verifica-se uma inconstância entre valores muito baixos seguidos de períodos com teores muito elevados de flúor, os quais podem ser atribuídos a deficiências do sistema de adição de flúor utilizado no município. A variação verificada entre os reservatórios reflete essa deficiência, uma vez que a água armazenada naqueles mais distantes corresponde à água tratada em um momento diferente daquela coletada imediatamente próxima às ETAs.

Estudos apontaram os vários fatores responsáveis pela oscilação na concentração ótima de fluoretos, destacando entre eles a carência de pessoal devidamente treinado para fazer o controle interno, problemas no equipamento hidráulico ou variações no fluxo de água (vazão) ao longo da rede de distribuição da cidade (Lima et al., 2004; Ferreira et al., 1999; Modesto et al., 1999; Narvai 2001). De acordo com Saliba et al. (2006) municípios de pequeno e médio porte podem ter dificuldades em realizar o controle da adição de flúor nas águas de abastecimento público em função da falta de infra-estrutura laboratorial e técnica.

Deve-se ainda considerar as possíveis limitações do método colorimétrico, aqui utilizado para a aferição dos teores de flúor nas amostras, o qual está mais sujeito a erros de leitura do que o do eletrodo. Dessa forma, os resultados relatados podem ser considerados como indícios preliminares, estando em curso a continuidade 
desta pesquisa, cujas amostras, coletadas por período similar, serão analisadas pelo método eletrométrico.

Os resultados desta pesquisa, bem como daqueles registrados na literatura, apontando irregularidades nos teores de fluoretos, reforçam a recomendação de Narvai (2001) sobre a necessidade da criação e manutenção de mecanismos de controle da concentração de flúor nas águas de abastecimento público. Oficializar um programa de heterocontrole da concentração de flúor na água é uma medida essencial para garantir os benefícios do método com segurança em relação à fluorose (Ministério da Saúde, 1999).

\section{Conclusões}

Verificou-se uma inconstância nos teores de fluoretos nas amostras coletadas predominando valores acima dos aceitáveis para o município. Este fato aponta para a necessidade de melhoria do controle operacional e uma política de vigilância sanitária que controle de forma satisfatória a sua execução.

\section{Agradecimentos}

Ao PIBIC/CNPQ, aos técnicos da SANEPAR de Ponta Grossa e ao Aurélio Rodrigues e Edelor Inglês da Luz (técnicos do laboratório de higiene social e análise de água da UEPG).

\section{REFERÊNCIAS}

1. BALDANI, M. H.; VASCONCELOS, A. G. G.; ANTUNES, J. L. F. Associação do Índice CPO-D com indicadores socioeconômicos e de provisão de serviços odontológicos no Estado do Paraná, Brasil. Caderno de Saúde Pública, Rio de Janeiro, v. 20, n.1, p. 143-52. 2004.

2. BIGHETTI, F. T. Fatores associados à fluorose dentária em área com água fluoretada. 2005. 221f. (Doutorado em Saúde Pública) - Faculdade de Saúde Pública, Universidade de São Paulo, São Paulo, 2005.

3. BRASIL. Lei n⿳ 6050, de 24 de maio de 1974. Dispõe sobre a obrigatoriedade da fluoretação das águas em sistemas de abastecimento. Disponível em: http://dtr2004.saude.gov.br/ dab/saudebucal/legislacao/lei6050_24_05_74.pdfAcesso em: 26 jan. 2006.

4. BRASIL. Ministério da Saúde. Portaria no 635, de 25 de dezembro de 1975. Estabelece os critérios e teores para adição do íon fluoreto. Disponível em: http://dtr2004.saude.gov.br/ dab/saudebucal/legislacao/portaria635_26_12_75.pdfAcesso em: 26 jan. 2006.

5. BROWNE, D.; WHELTON, H.; O’MULLANE, D. Fluoride metabolism and fluorosis. Journal of Dentistry, Bristol, v. 33, p. 177-86, Mar. 2005.

6. BURT, B. A. Fluoridation and social equity. J Public Health Dent, 2002; 62: 195-200.

7. CANGUSSU, M. C. T. et al. A fluorose dentária no Brasil: uma revisão crítica. Caderno de Saúde Pública, Rio de Janeiro, v. 18, n. 1, p. 07-15, jan./fev. 2002.

8. CENTERS FOR DISEASE CONTROLAND PREVENTION. Recomendaciones sobre el uso de fluoruros para prevenir y controlar la caries dental en los Estados Unidos. Revista Panamericana de Salud Publica, Washington, v. 11, n. 1, p. 59-66, enero 2002.

9. CURY, J. A. Fluoretação da água: benefícios, riscos e sugestões. Revista Odontológica do Brasil Central, São Paulo, v. 2, n. 5, p. 32-33, dez. 1992.

10. CURY, J. A. Uso do flúor e controle da cárie como doença. In: BARATIERI, L. N. et al. Odontologia restauradora: fundamentos e possibilidades. São Paulo: Ed. Santos, 2001. cap. 1, p. 33-68.

11. DANTAS, N. L. Sistema de vigilância dos teores de flúor na água de abastecimento público de Curitiba. Divulgação, $n$. 13, p. 70-82, jul. 1996.

12. DITTERICH, R. G. Prevalência e autopercepção de fluorose dentária em escolares de 12 anos residentes no município de Ponta Grossa-PR. 2006. 158f. Dissertação (Mestrado em Odontologia) - Faculdade de Odontologia, Universidade Estadual de Ponta Grossa, Ponta Grossa, 2006.

13. FERREIRA, H. C. G. et al. Avaliação do teor de flúor na água de abastecimento do município de Vitória-ES. Revista da Associação Paulista de Cirurgiões-dentistas, São Paulo, v. 53, n. 6, p.—, nov./dez. 1999.

14. FRAZÃO, P. et al. Fluorose dentária: comparação de dois estudos de prevalência. Cadernos de Saúde Pública, Rio de Janeiro, v. 20, n. 4, p.1050-8, jul./ago. 2004.

15. (IBGE) Instituto Brasileiro de Geografia e Estatística. IBGE - Cidades. Disponível na internet em: www.ibge.gov.br/ cidadesat/default.php. (acesso em 23/08/2007).

16. JONES CM, TAYLOR GO, WHITTLE JG, EVANS D, TROTTER DP. Water fluoridation, tooth decay in 5 year olds, and social deprivation measured by the Jarman score: analysis of data from British dental surveys. Br Med J 1997; 315: $514-$ 518.

17. JONES CM, Worthington $\mathrm{H}$. Water fluoridation, poverty and tooth decay in 12-year-old children. J Dent 2000; 28: 389393 
18. KOZLOWSKI, F. C. Fluorose dentária em dois grupos etários no município de Ponta Grossa-PR. 2004. 190f. Tese (Doutorado em Odontologia) - Faculdade de Odontologia de Piracicaba, Universidade Estadual de Campinas, Piracicaba, 2004.

19. KOZLOWSKI, F. C.; PEREIRA, A. C. Métodos de utilização do flúor sistêmico In: PEREIRA, A. C. et al. Odontologia em saúde coletiva: planejando ações e promovendo saúde. Porto Alegre: Artmed, 2003. cap. 14, p. 265-74.

20. LIMA, B. O.; CURY, J. A. Ingestão de flúor por crianças pela água e dentifrício. Revista de Saúde Pública, São Paulo, v. 35, n. 6, p. 576-81, dez. 2001.

21. LIMA, F. G. et al. Vinte e quatro meses de heterocontrole da fluoretação das águas de abastecimento público de Pelotas, Rio Grande do Sul, Brasil. Caderno de Saúde Pública, Rio de Janeiro, v. 20, n. 2, p. — mar./abr. 2004.

22. MAIA, L. C. et al. Controle operacional da fluoretação da água de Niterói, Rio de Janeiro, Brasil. Caderno de Saúde Pública, Rio de Janeiro, v. 19, n. 1, p. 661-7, jan./fev. 2003.

23. MALTZ, M. et al. Prevalência de fluorose em duas cidades brasileiras, uma com água artificialmente fluoretada e outra com baixo teor de flúor, em 1987 e 1997/98. Revista da Faculdade de Odontologia da UFRGS, Porto Alegre, v. 42, n. 2, p. 51-55, dez. 2000

24. MASCARENHAS, A. K. Risk factors for dental fluorosis: A review of the recent literature. Pediatric Dentistry, Chicago, v. 22, n. 4, p. 269-277, July/Aug. 2000.

25. MODESTO, A. et al. Avaliação da concentração de fluoreto na água de abastecimento público do município do Rio de Janeiro. Revista Brasileira de Odontologia, Rio de Janeiro, v. 56, n. 5, p.—, set./out, 1999.

26. MOYSÉS, S. J. et al. Fluorose dentária: ficção epidemiológica? Revista Panamericana de Salud Publica, Washington, v. 12, n. 5, p. 339-46, nov. 2002.

27. MURRAY, J. J. O uso correto de fluoretos na saúde pública. São Paulo: Ed. Santos, 1992.

28. NARVAI, P. C. Cárie dentária e flúor: uma relação do século XX. Ciência \& Saúde Coletiva, Rio de Janeiro, v. 5, n. 2, p. 381$92,2000$.

29. NARVAI, P. C. Vigilância Sanitária da fluoretação das águas de abastecimento público no município de São Paulo, Brasil, no período de 1990 - 1999. Tese de Livre Docência. São Paulo: Faculdade de Saúde Pública, USP. 2001.

30. OLIVEIRA, A. N. C.;AMARAL, C. R. F. L.; PEREIRA, C. R. Fluorose dentária: uma revisão bibliográfica. Revista da ABO Nacional, Porto Alegre, v. 8, n. 2, p. 92-7, abr./maio. 2000.

31. ORGANIZACIÓN MUNDIAL DE LA SALUD. Los fluoruros y la salud bucodental: informe de um Comitê de Expertos de la OMS en el Estado de Salud Bucodental y el Uso de Fluoruros. Genebra. 1994. 44 p. (Serie de informes técnicos: 846).

32. ORGANIZAÇÃO MUNDIAL DA SAÚDE. Levantamento epidemiológico básico de saúde bucal. 4. ed. São Paulo: Ed. Santos, 1999.

33. PEREIRA, A. C. et al. Prevalência de cárie e fluorose dentária em escolares de cidades com diferentes concentrações de flúor na água de abastecimento. Revista Brasileira de Odontologia em Saúde Coletiva, Florianópolis, v. 2, n. 1, p. 34-39, jan. 2001.

34. PINTO, V. G. Saúde Bucal: Odontologia social e preventiva, 3 ed., São Paulo: Editora Santos, 1992.

35. RAMIRES, I. et al. Heterocontrole da fluoretação da água de abastecimento público em Bauru, SP, Brasil. Rev. Saúde Pública, Out 2006, v.40, n..5, p.883-889.

36. RAMOS, N. B. et al. Riscos de fluorose dentária em crianças com 2, 3 e 4 anos de idade, que consomem água fluoretada, apresentando concentrações de 0,1 a 0,7 ppm de íons fluor, e utilizam creme dental fluoretado. Revista do Instituto de Ciências da Saúde, São Paulo, v. 22, n. 2, p. 149-54, abr./jun. 2004.

37. RIBAS, T. R. C. et al. Avaliação da fluorose dentária em escolares do Colégio Vicente de Paulo, situado na Zona Leste do município de São Paulo. Revista de Odontologia da Universidade de Santo Amaro, São Paulo, v. 4, n. 2, p.62-7, jul./ dez. 1999.

38. SALIBA, N. A; MOIMAZ, S. A. S; TIANO, A. V. P. Fluoride level in public water supplies of cities from the northwest region of São Paulo State, Brazil 14(5):346-350, Sept.-Oct. 2006. J appli oral sci.

39. SCHNEIDER FILHO, D. A. et al. Cadernos de Saúde Bucal 2 - fluoretação da água: como fazer a vigilância sanitária? Rio de Janeiro: Rede Cedros. 1992. 28 p.

40. SILVA, J. S.; VAL, C. M.; COSTA, J. N.; MOURA, M. S.; SILVA, T.A. E.; SAMPAIO, F. C. Heterocontrole da fluoretação das águas em três cidades no Piauí, Brasil / Monitoring water fluoridation in three cities in Piauí State, Brazil Caderno de Saúde Pública 23(5):1083-1088, maio 2007.

41. SLADE, G. D. et al. Influence of exposure to fluoridated water on socioeconomic inequalities in children's caries experience. Community Dent Oral Epidemiol 1996; 24: 89-100.

42. TOASSI, R. F. C.; KUHNEN, M.; CISLAGHI, G. A.; BERNARDO, J. R. Heterocontrole da fluoretação da água de abastecimento público de Lages, Santa Catarina, Brasil / External control of fluoride levels in the public water supply in Lages, Santa Catarina State, Brazil Ciência \& Saúde Coletiva, 12(3):727-732, maio-jun. 2007. 